\title{
Proper Treatment Option for Small Rectal Neuroendocrine Tumors Using Precut Endoscopic Mucosal Resection
}

\author{
Seun Ja Park \\ Department of Internal Medicine, Kosin University Gospel Hospital, Kosin University College of Medicine, Busan, Korea
}

See "Efficacy of Precut Endoscopic Mucosal Resection for Treatment of Rectal Neuroendocrine Tumors" by Hoonsub So, Su Hyun Yoo, Seungbong Han, et al., on page 585-591.

In the past, rectal neuroendocrine tumors (NETs) were uncommon neuroendocrine neoplasms. Recently, the incidence of rectal NETs has increased as the number of screening colonoscopies has increased. Thus, rectal NETs are one of the most common NETs found in the gastrointestinal tract. In a recent Korean single-center study, the same results showed that the most frequent primary site of NETs in the gastrointestinal tract is the rectum $(79.8 \%){ }^{1}$

Most rectal NETs covered with yellowish normal mucosa are small, localized, and mucosal or submucosal in location. A large prospective study of prognostic features of rectal NETs in 347 patients reported that the risk factors of metastasis include increasing tumor size, increased mitotic rate, lymphovascular invasion, and depression or ulceration observed macroscopically on endoscopy. ${ }^{2}$ None of the patients with a tumor size of $\leq 10 \mathrm{~mm}$ had metastatic disease. The metastatic potential of rectal NETs of $<10 \mathrm{~mm}$ in size has been reported to be as low as $2 \%$, increasing to $10 \%-15 \%$ in tumors ranging from $1-2 \mathrm{~cm}$ and $60 \%-80 \%$ in tumors of $>2.0 \mathrm{~cm}$ in size.,

If the size of the rectal NET is smaller than $10 \mathrm{~mm}$, the risk of lymph node of distant metastasis is low. Thus, in these cases, it can generally be treated by conventional polypecto-

Received: October 30, 2017 Accepted: November 13, 2017

Correspondence: Seun Ja Park

Department of Internal Medicine, Kosin University Gospel Hospital, Kosin University College of Medicine, 262 Gamcheon-ro, Seo-gu, Busan 49267, Korea Tel: +82-51-990-5061, Fax: +82-51-990-3046, E-mail: parksj@ns.kosinmed.or.kr

(cc) This is an Open Access article distributed under the terms of the Creative Commons Attribution Non-Commercial License (http://creativecommons.org/ licenses/by-nc/3.0) which permits unrestricted non-commercial use, distribution, and reproduction in any medium, provided the original work is properly cited. my, ${ }^{3-5}$ especially if they do not have other risk factors such as increased mitotic rate or lymphovascular invasion. However, conventional polypectomy has been shown to be an ineffective treatment method for rectal NETs, as most of these lesions extend into the submucosa. Some studies have reported that the complete resection rate of conventional polypectomy was as low as $20 \%-30 \%{ }^{5,6}$ To increase the likelihood of complete pathological resection rate, modified endoscopic mucosal resection (EMR) techniques such as EMR using a transparent cap, EMR with a ligation device, and endoscopic submucosal resection with a ligation device are needed. All these methods that use suction can make a pseudostalk before resection. ${ }^{7-10}$ Another important advanced method to achieve a negative resection margin is endoscopic submucosal dissection (ESD). ESD is a resection technique for early gastric cancer. Its use has increased in colorectal tumors and NETs. The advantage of ESD is to achieve complete en bloc resection regardless of tumor size. The disadvantage of ESD is that it is time-consuming and complicated. ${ }^{11}$ Resection time was longer in the ESD group than that in the EMR group (11.4 \pm 3.7 min vs. $4.2 \pm 3.2$ $\min , p<0.001){ }^{11}$

In this issue of Clinical Endoscopy, So et al. proposed a precut endoscopic mucosal resection (EMR-P) method for the treatment of rectal NETs, which was performed as follows: After submucosal injection, circumferential incision/precutting was performed using the tip of the snare to cut along a 2-mm margin outside the tumor. ${ }^{12}$ Subsequently, the snare was securely positioned in the cut groove and tightened, and the tumor was resected using electrical current. En bloc and complete resections were achieved in $71(98.6 \%)$ and 67 patients 
(93.1\%), respectively. The mean time required for resection was $9.0 \pm 5.6 \mathrm{~min}$. Immediate and delayed bleeding developed in $6(8.3 \%)$ and 4 patients $(5.6 \%)$, respectively. ${ }^{12}$

Compared with previous EMR-P $\mathrm{P}^{13,14}$ or modified EMR or ESD, the treatment method described in this study has the advantage of the use of a snare tip rather than specialized endoknives for precutting or a band, cap, or two-channel scope. Use of the snare tip reduced the time and cost of this procedure because additional accessories did not have to be introduced and withdrawn before snaring. ${ }^{12}$

In conclusion, the EMR-P method is one such modification to achieve high negative pathologic resection rate and a short procedure time without additional costs.

\section{Conflicts of Interest}

The author has no financial conflicts of interest.

\section{REFERENCES}

1. Lim CH, Lee IS, Jun BY, et al. Incidence and clinical characteristics of gastroenteropancreatic neuroendocrine tumor in Korea: a single-center experience. Korean J Intern Med 2017;32:452-458.

2. Chablaney S, Zator ZA, Kumta NA. Diagnosis and management of rectal neuroendocrine tumors. Clin Endosc 2017;50:530-536.

3. Park $\mathrm{CH}$, Cheon JH, Kim JO, et al. Criteria for decision making after endoscopic resection of well-differentiated rectal carcinoids with regard to potential lymphatic spread. Endoscopy 2011;43:790-795.

4. Al Natour RH, Saund MS, Sanchez VM, et al. Tumor size and depth predict rate of lymph node metastasis in colon carcinoids and can be used to select patients for endoscopic resection. J Gastrointest Surg 2012;16:595-602.

5. Onozato Y, Kakizaki S, Iizuka H, Sohara N, Mori M, Itoh H. Endoscopic treatment of rectal carcinoid tumors. Dis Colon Rectum 2010;53:169176.

6. Son HJ, Sohn DK, Hong CW, et al. Factors associated with complete local excision of small rectal carcinoid tumor. Int J Colorectal Dis 2013;28:57-61.

7. Saito Y, Otake Y, Sakamoto T, et al. Indications for and technical aspects of colorectal endoscopic submucosal dissection. Gut Liver 2013;7:263269.

8. Choi HH, Kim JS, Cheung DY, Cho YS. Which endoscopic treatment is the best for small rectal carcinoid tumors? World J Gastrointest Endosc 2013;5:487-494

9. Park SJ. Tips and tricks for better endoscopic treatment of colorectal tumors: usefulness of cap and band in colorectal endoscopic mucosal resection. Clin Endosc 2013;46:492-494.

10. Kim HH, Park SJ, Lee SH, et al. Efficacy of endoscopic submucosal resection with a ligation device for removing small rectal carcinoid tumor compared with endoscopic mucosal resection: analysis of 100 cases. Dig Endosc 2012;24:159-163.

11. Park HW, Byeon JS, Park YS, et al. Endoscopic submucosal dissection for treatment of rectal carcinoid tumors. Gastrointest Endosc 2010;72:143-149.

12. So H, Yoo SH, Han S, et al. Efficacy of precut endoscopic mucosal resection for treatment of rectal neuroendocrine tumors. Clin Endosc 2017;50:585-591.

13. Huang J, Lu ZS, Yang YS, et al. Endoscopic mucosal resection with circumferential incision for treatment of rectal carcinoid tumours. World J Surg Oncol 2014;12:23.

14. Cheung DY, Choi SK, Kim HK, et al. Circumferential submucosal incision prior to endoscopic mucosal resection provides comparable clinical outcomes to submucosal dissection for well-differentiated neuroendocrine tumors of the rectum. Surg Endosc 2015;29:1500-1505. 